\title{
Increased detection of precancerous cervical lesions with adjunctive dynamic spectral imaging
}

\author{
This article was published in the following Dove Press journal: \\ International Journal of Women's Health \\ 28 September 2017 \\ Number of times this article has been viewed
}

\author{
Sara A DeNardis' \\ Philip T Lavin ${ }^{2}$ \\ Jeff Livingston ${ }^{3}$ \\ William R Salter ${ }^{4}$ \\ Nanette James-Patrick ${ }^{5}$ \\ Emmanouil Papagiannakis ${ }^{6}$ \\ Christopher G Olson ${ }^{7}$ \\ Lori Weinberg ${ }^{8}$ \\ 'Department of Obstetrics/ \\ Gynecology, University of Central \\ Florida, Orlando, FL, USA; ${ }^{2}$ Boston \\ Biostatistics Research Foundation, \\ Framingham, MA, USA; ${ }^{3}$ MacArthur \\ OB/GYN, Irving, TX, USA; ${ }^{4}$ Advanced \\ ObGyn Associates, Richardson, TX, \\ USA; ${ }^{5}$ Southwest Women's Healthcare \\ Associates, Olympia Fields, IL, USA; \\ ${ }^{6}$ DYSIS Medical, Edinburgh, UK; \\ ${ }^{7}$ Women's Center for Health, \\ Naperville, IL, USA; ${ }^{8}$ Department of \\ Obstetrics/Gynecology, Advocate \\ Illinois Masonic Medical Center, \\ Chicago, IL, USA
}

Correspondence: Sara A DeNardis Department of Obstetrics/Gynecology, University of Central Florida, 720 West Oak Street, Suite 20I, Kissimmee, FL 3474I, USA

Email saradenardis@icloud.com
Objective: To validate, in US community-based colposcopy clinics, previous reports of increased detection of high-grade cervical intraepithelial neoplasia (CIN2+) with biopsies selected using dynamic spectral imaging (DSI) mapping after standard colposcopy.

Study design: Cross-sectional observational study of 26 colposcopists across nine clinics recruiting consecutive colposcopy patients. Standard assessment with biopsy selections was completed before seeing the DSI map which was subsequently interpreted and used for additional biopsies per clinical judgment. Primary measure was the number of women with CIN2+ detected by DSI-assisted biopsies, over those detected by standard colposcopy biopsies.

Results: A total of 887 women were recruited. After exclusions, 881 women and 1,189 biopsies were analyzed. Standard biopsy detected 78 women with CIN2+ and DSI-assisted biopsies another 34 , increasing the detection rate from $8.85 \%$ to $12.71 \%(p=0.00016)$. This was achieved with $16.16 \%$ of DSI-assisted biopsies finding CIN2+ compared to $13.24 \%$ for the preceding standard biopsies. For secondary specificity analysis, 431 women had only $<$ CIN2 in standard biopsy/ies, a $48.92 \%$ rate, while 131 women who underwent no standard biopsy/ies had DSIassisted biopsy/ies which were all $<\mathrm{CIN} 2$, a $35.22 \%$ rate $(p<0.0001)$.

Conclusion: The largest study, to date, of DSI used in colposcopy confirms previously reported increased detection of CIN2+, across multiple US community-based clinics. Based on the improved efficiency of the DSI-assisted biopsies, this increase suggests an improved diagnostic capacity achieved with DSI and cannot be explained solely by the taking of additional biopsies.

Keywords: biopsy, cervix uteri, CIN2+, colposcopy, dynamic spectral imaging

\section{Introduction}

Colposcopy with biopsy is critical for cervical cancer prevention programs, as it is used to diagnose precancerous cervical intraepithelial neoplasia (CIN) in patients selected by screening with cytology and/or human papillomavirus (HPV) testing. ${ }^{1}$ Colposcopy suffers from low sensitivity and high subjectivity ${ }^{2-7}$ and taking more biopsies increases detection, ${ }^{8,9}$ so implementation approaches supporting multiple biopsies, ${ }^{10}$ directed ${ }^{11}$ or standard four-quadrant with random biopsies, ${ }^{12,13}$ have been proposed. However, as evidence from this setting is scarce, it is uncertain to what extent these practices are widely adopted by community colposcopists (recently presented data indicates the contrary $)^{14}$ and the applicability of academic institution-based study findings to community-based practice is questioned..$^{15}$ Furthermore, even with multiple biopsies, comparisons to same-day treatment specimens show that biopsies often miss the area of worst pathology. ${ }^{16,17}$ Therefore, accurate localization of precancerous cervical lesions challenges colposcopists. 
With the adoption of HPV testing which detects smaller CIN3 lesions, ${ }^{5}$ the expected drop in colposcopy volumes, ${ }^{18}$ the introduction of HPV testing for primary screening in the USA, ${ }^{19}$ as well as the difficulty of detecting high-grade disease in smaller lesions which do not present with classic patterns,${ }^{17}$ improving the sensitivity of colposcopy, and supporting colposcopic assessments becomes even more important.

Among the different criteria evaluated during colposcopy, cervical acetowhitening is the most sensitive for CIN2+. ${ }^{20}$ Dynamic spectral imaging (DSI) calculates a color map which quantifies the degree of acetowhite changes over the cervix to introduce objectivity and assist assessment and biopsy placement. International, academic institution-based trials demonstrated that the DSI map increases sensitivity for CIN2+ significantly. ${ }^{21-24}$ These research studies were performed in controlled settings, where colposcopists had to follow the most severe DSI indications for biopsy and also included random biopsies to reduce verification bias. To date, the effectiveness of DSI in the detection of CIN2+ in routine practice and wider US settings has not been evaluated, except for a single-colposcopist observational study from Spain which confirmed an increased sensitivity. ${ }^{25}$

The objective of this study is to examine how the, asintended, use of the adjunctive DSI map impacts colposcopy results in a large cohort of patients having colposcopy across multiple community-based clinics in the USA, and analyze the specific contribution of DSI-identified biopsies. To this end, this manuscript analyzes the additional number of patients found with CIN2+ by use of the DSI map for biopsy after biopsy selection by standard assessment is complete. Additionally, it compares this to the number of biopsied patients and the number of biopsies taken, to investigate whether any increase in CIN2+ detection results from an improved diagnostic ability with the DSI map rather than from simply taking additional biopsies.

\section{Materials and methods}

\section{Registration: ClinicalTrials.gov (NCT 02185599).}

The IMPROVE-COLPO study is a multi-center, observational, cross-sectional study in women having colposcopy based on current US guidelines. ${ }^{1,19}$ The study was approved by a central Institutional Review Board (E\&I Review Services, Independence, MO, USA) and all patients signed informed consent prior to any procedures. Participating colposcopists included gynecologic oncologists, obstetrician-gynecologists, nurse practitioners, and physician assistants. Colposcopists were adequately trained on using the device prior to recruiting patients. Each participating facility recruited eligible women from their consecutive routine colposcopy examinations performed with the DSI digital colposcope. This is an analysis of data from the first nine sites that completed participation, focusing on the colposcopic examinations with the DSI colposcope, with the objective to evaluate the additional high-grade precancerous lesions found by DSI-assisted biopsies in routine practice.

Inclusion criteria were age $\geq 21$ years old and an abnormal screening test result. ${ }^{1,19}$ Although guidelines recommend against it, HPV testing is frequently used to co-test women $<29$, so these were included also with a combination of atypical squamous cells of undermined significance (ASC-US) and HPV+ result. Women 21-24 were also included with a single low-grade squamous intraepithelial lesion result. Women undergoing colposcopy for no specified indication or after a single ASC-US or a single HPV+ result (unless they were $>25$ and had HPV16/18 from primary screening $)^{19}$ were excluded. Other exclusions were for known pregnancy, HIV infection or AIDS, previous hysterectomy, and for receiving (or having previously received) radiation treatment or chemotherapy for cancers concurrent with cervical disease.

The study device, DSI colposcope (DYSIS, DYSIS Medical, Edinburgh, UK), is a commercial digital colposcope offering magnification, green and enhanced-contrast filters, annotation and overlay of biopsy selections for guidance, dynamic playback for image comparisons during the examination, and DSI mapping. ${ }^{23}$ The DSI is a method to quantify and map the cervical acetowhitening with a procedure that includes application of acetic acid with an integrated applicator system to standardize quantity, coverage of the cervix, and timing. During the development of acetowhitening, and while the colposcopist is assessing it visually, the system collects images of the cervix and compares them to a baseline (ie, pre-acetic acid) image. It then extracts the intensity and temporal profile of the acetowhitening over the different areas of the cervix to calculate the color-coded DSI map which can assist assessment and biopsy placement (Figure 1). This process takes place in the background and the map is available for review within the 2-3 minutes that it typically takes the acetowhitening to fully evolve, thereby not adding time to the colposcopic examination.

During the study, the device was used according to the indications for use cleared by the US Food and Drug Administration, whereby the DSI map is an adjunct to colposcopy, splitting the examination into two phases: the first phase is 

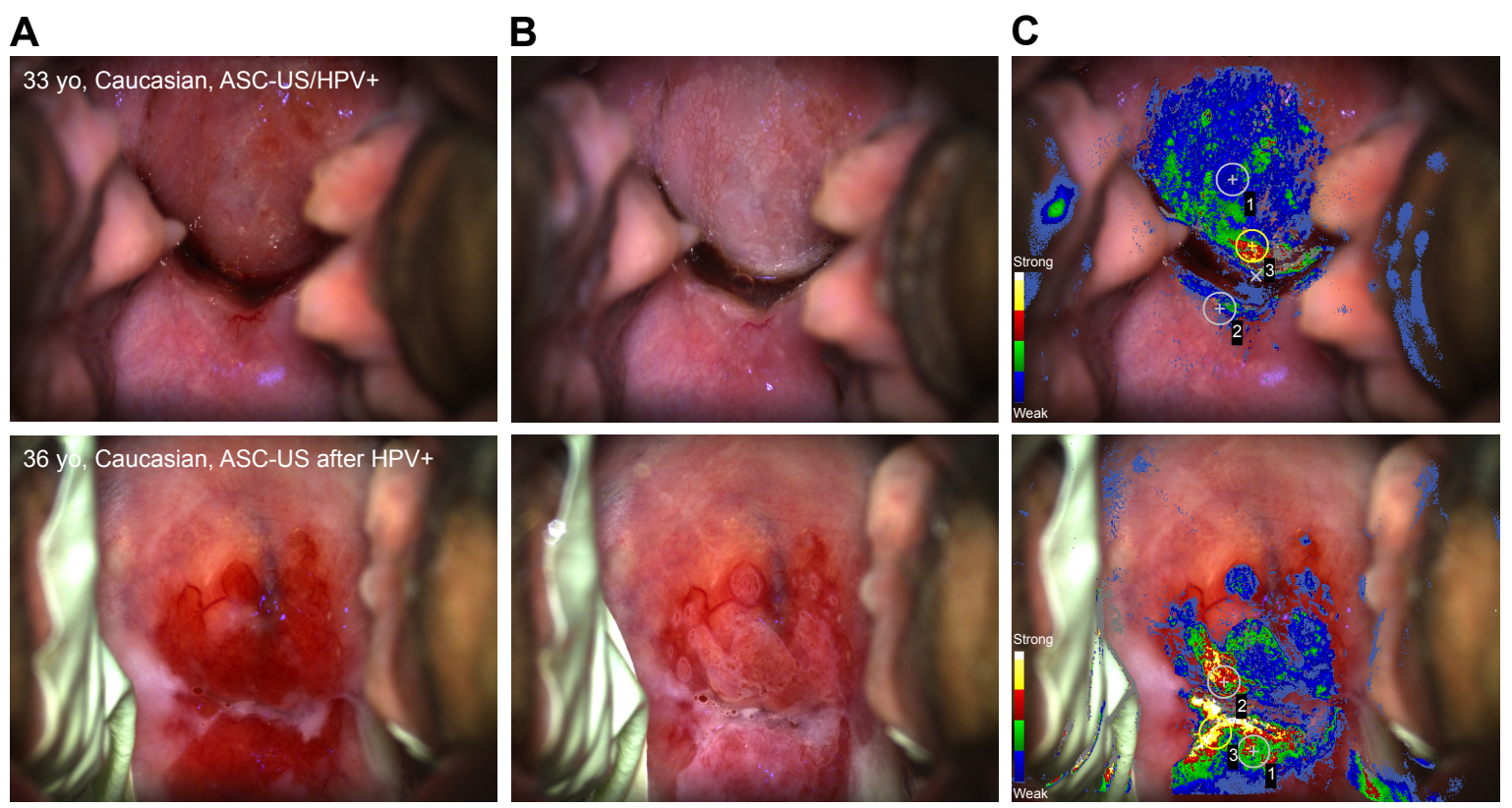

Figure I Example of cervical images from two patients, with DSI map and biopsy annotations.

Notes: (A) Before acetic acid application. (B) Two minutes after acetic acid application. (C) DSI map and biopsy annotations. Gray: standard biopsy selected before the DSI map; Yellow: selected with the DSI map. Top row: two sites (I, 2) were selected for biopsy initially. A third site was selected at an area highlighted by red/yellow DSI colors (3). The two standard-directed biopsies found CINI and the DSI-assisted biopsy found CIN2. Without the DSI-assisted biopsy the high-grade lesion would have been missed. Bottom row: two sites (I, 2) were selected for biopsy initially. An additional area highlighted by red/yellow DSI colors was also biopsied (3). The two standarddirected biopsies found CIN2 and the DSI-assisted biopsy found CIN3, helping to capture the worst pathology area.

Abbreviations: ASC-US, atypical squamous cells of undermined significance; CIN, cervical intraepithelial neoplasia; DSI, dynamic spectral imaging; HPV, human papillomavirus.

the thorough standard visualization of the cervix to assess morphology and acetowhitening, with biopsy selections indicated on the software interface (the DSI map is not yet accessible), and the second phase is further assessment and biopsy selection with the DSI map. Biopsies identified in the first phase should never be cancelled because of the DSI map. When no biopsies were indicated during the first phase, clinicians had to explicitly confirm and document that on the software interface. All clinical decisions were the responsibility of the colposcopist: whether to biopsy a patient, number of biopsies to be taken, where to biopsy. So, after the selection of biopsies based on standard assessment, the DSI map was interpreted and used at the colposcopists' discretion and was followed/overridden for biopsy as considered appropriate for each patient. Random biopsy and endocervical sampling were performed per clinical judgment. For each woman, the study collected basic demographic information, the number of biopsies taken, whether endocervical sampling was performed, and all relevant histological outcomes. Each biopsy taken was indicated on the study forms as "Standard", "DYSIS" or "Random" to allow tracking of histopathology findings. Random biopsy was defined as a biopsy from an area considered normal. Results were collected at the biopsy-level, except for patients with multiple biopsies who had all samples collected in the same jar and processed together. Histopathology readings were performed at the laboratories collaborating with the participating facilities, following routine practice, and were the gold standard for analyses.

The main outcome measures were the number of women with detected CIN2+ (CIN2, CIN3, [adeno] carcinoma in situ, and invasive carcinomas), the number of women biopsied, and the number of biopsies taken. CIN2 was used as the threshold, because this is the standard outcome which drives management decisions. ${ }^{26}$

This manuscript compares the findings of standard colposcopic biopsy (first phase of the examination) and DSIassisted biopsy (second phase) using the patient as her own control for comparisons. A comparison of colposcopy with DSI to a control group of colposcopy with standard methods will be presented separately.

We conducted subject-level and biopsy-level analyses, and did not consider findings of endocervical sampling or treatments. Sensitivity and specificity could not be directly calculated because, without extensive biopsying, excisional treatment on every patient or longitudinal follow-up, the number of subjects with undetected disease is unknown. On the subject-level analysis, we determined the CIN2+ detection 
rate for each phase of the examination. For the first phase, this is the number of women with at least one CIN2+ biopsy during standard colposcopy, divided by the total population. For the second phase, the detection rate is the number of women who had no biopsies or $<$ CIN2 biopsies during standard colposcopy (first phase) and had at least one CIN2+ DSI-assisted biopsy, divided by the number of women who were not found to have CIN2+ during the first phase. With $>800$ subjects not found to be CIN2+ during the first phase of the examination, the sample size was adequate to reject the $2 \%$ null hypothesis for the incremental gain in the detection rate vs a $4 \%$ alternative hypothesis, with $90 \%$ power and two-sided 5\% Type-I error. The detection rates were also calculated for CIN3+.

We also determined the rates of patients with only negative $(<\mathrm{CIN} 2)$ biopsies. For the first phase, this is the number of women with only $<$ CIN2 standard-directed biopsies over the total number of women. For the second phase, it is the number of women who had no biopsy taken during the first phase but had DSI-assisted biopsy/ies (second phase) which were all $<$ CIN2, divided by the number of women who had no biopsy during the first phase.
To evaluate the effectiveness of using DSI for biopsy selection in detecting high-grade CIN, and compare it to the preceding standard-directed biopsies, we calculated, the number needed to detect (NND) and the positive predictive value (PPV) of biopsy for each phase of the examination. NND is the ratio of the number of women with only $<$ CIN2 on their biopsy/ies, over the number of women found with CIN2+. The PPV is the number of CIN2+ biopsies divided by the number of biopsies taken.

\section{Results}

The study recruited 887 women (Figure 2) who were examined by 26 colposcopists across the nine sites, between September 2014 and May 2016. Six women were excluded from all analyses, the reasons being age $<21(n=2)$, previous hysterectomy $(\mathrm{n}=2)$, not meeting referral inclusion criteria $(n=2)$. No study or study-device related adverse events were reported. Table 1 describes the characteristics of the 881 women included in the analyses; their median age was 33.0 years and most $(91.4 \%)$ were privately insured, which could be indicative of a population of lower-risk ${ }^{27}$ served in the US community compared to institution-based clinics.

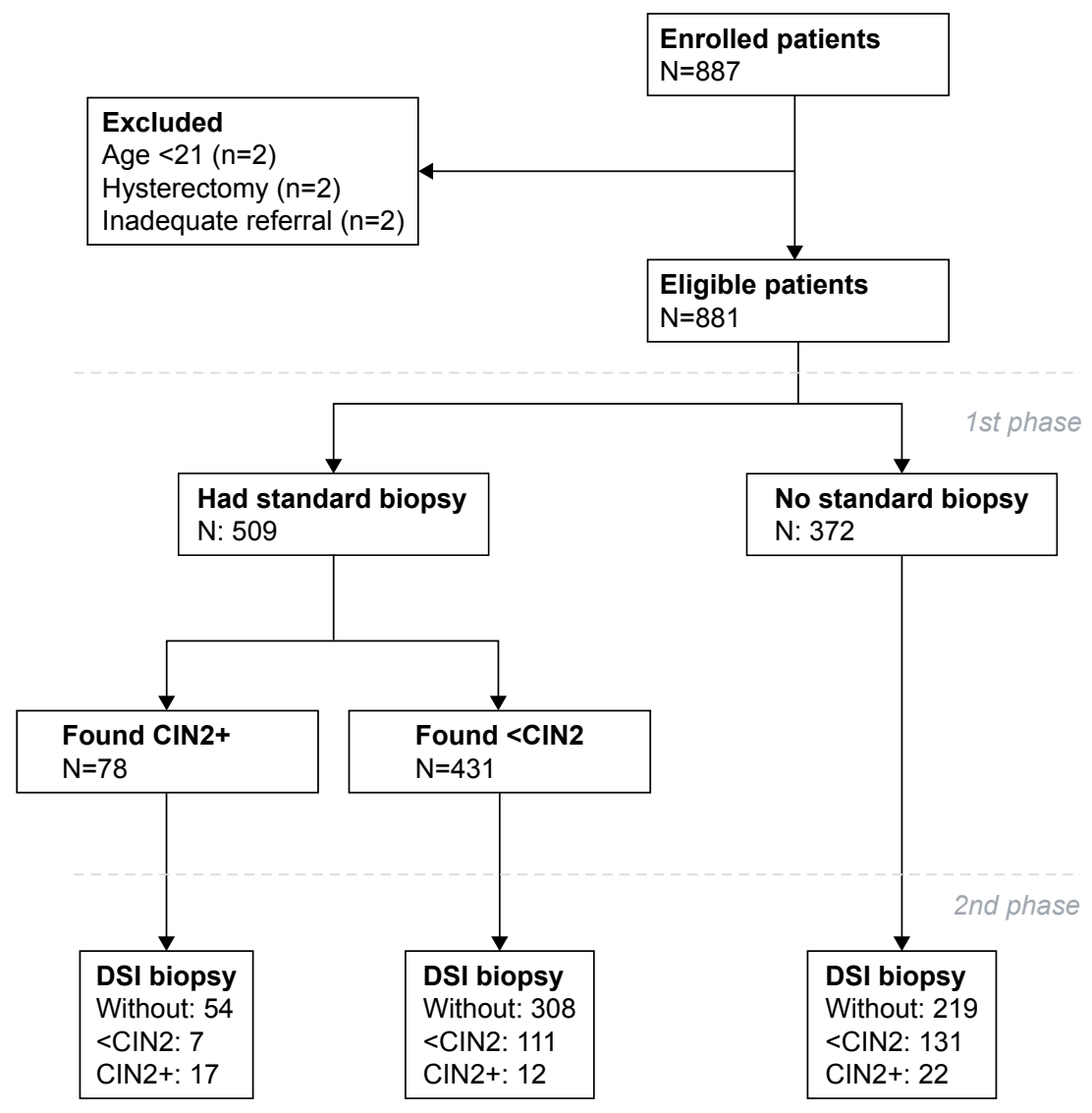

Figure 2 Study flow diagram (data are number of patients).

Abbreviations: CIN, cervical intraepithelial neoplasia; DSI, dynamic spectral imaging. 
Table I Patient baseline characteristics

\begin{tabular}{ll}
\hline Characteristic & N (\%) \\
\hline Included patients & $88 \mathrm{I}$ \\
Age (years) & \\
$\quad$ Mean (range) & $35.9(2 \mathrm{I}-80)$ \\
$\quad$ Median (25\%-75\% percentile) & $33.0(27-43)$ \\
Pre-menopausal & $782(88.8)$ \\
Insurance status & \\
$\quad$ Private & $805(91.4)$ \\
Medicare & $16(1.8)$ \\
Medicaid/other & $50(5.7)$ \\
Uninsured & $9(1.0)$ \\
Race/ethnicity & \\
White/Caucasian & $527(59.8)$ \\
Black/African-American & $142(16.1)$ \\
Asian & $43(4.9)$ \\
Hispanic & $149(16.9)$ \\
Other & $20(2.3)$ \\
Referral & \\
HSIL, ASC-H, AGC & $\mathrm{II}(12.5)$ \\
LSIL, ASC-US/HPV+, HPV + & $771(87.5)$ \\
\hline
\end{tabular}

Note: Missing values have been excluded.

Abbreviations: AGC, atypical glandular cells; ASC-H, atypical squamous cells (cannot exclude HSIL); ASC-US, atypical squamous cells of undermined significance; HPV, human papillomavirus; HSIL, high-grade squamous intraepithelial lesion; LSIL, low-grade squamous intraepithelial lesion.

Biopsy was obtained from 667 (75.71\%) women and endocervical sample from 615 (69.81\%), 94 of whom had no biopsy taken. Of the 1,189 biopsies taken, 808 were standard-directed biopsies (from 509 women), 365 were DSIassisted (from 300 women, 153 of whom had no standard biopsy) and 16 were random (from seven women, five of them without standard/DSI biopsy). The overall average number of biopsies per woman was 1.35 , and was 1.78 among the 667 who were biopsied. Among biopsied women, the average number of standard-directed biopsies was 1.21 and the average number of DSI-assisted biopsies was 0.55 . Colposcopic biopsy (any kind) detected 113 women with CIN2+. Six patients who had multiple biopsies, had their samples analyzed together, but none of them had CIN2+, so this has no effect on the analyses. Endocervical sampling found CIN2+ in 28 women; seven of these were additional to those found by biopsy (two from women without biopsies and five from women with only $<\mathrm{CIN} 2$ in their biopsies). Four invasive cervical cancers were found; one by multiple standard-directed biopsies, one by a DSI-assisted biopsy, and two by treatments following CIN3 in standard-directed biopsy. There were six cases of high-grade glandular lesions/ adenocarcinomas in situ, of which five were picked up by standard-directed biopsy/ies and one by treatment for a CIN3 lesion which was found by a DSI-assisted biopsy. The numbers of CIN2+ and CIN3+ women detected by each type of biopsy, stratified by age group, referral category, and number of biopsies taken per patient can be seen from Table 2. Due to small numbers, random biopsies were not considered further in the following section.

Standard-directed biopsy (first phase of the examination) detected 78 women with CIN $2+(8.85 \%$ detection rate, $95 \%$ CI: $7.06 \%-10.93 \%$ ) (Table 3). During the second phase, DSI-assisted biopsies detected 34 women with CIN2+ among the 803 not initially detected $(4.23 \%$ detection rate, $95 \%$ CI: $2.95 \%-5.87 \%$ ). These additional 34 detected women, 22 of whom had no standard biopsy indicated during the first phase, underwent 20 standard biopsies (all being $<\mathrm{CIN} 2$ ), and had 50 DSI-assisted biopsies (41 of them being CIN2+), a statistically significant difference in number of biopsies (20 vs 50, $p=0.0004$, exact binomial test) and in detection ( 0 vs $34, p<0.0001$, Fisher's exact test).

The detection rate including the DSI-assisted biopsies was $12.71 \%$ (95\% CI: $10.58 \%-15.09 \%)$, a $3.86 \%$ addition to the initial $8.85 \%$ rate, a significant difference (twosided $p=0.00016$, exact binomial test) which rejects the $2 \%$ null hypothesis (two-sided $p=0.0001$, exact binomial test) and a relative gain of $43.6 \%$ in women detected with CIN2+.

Detection rates were also calculated for CIN3+, a better surrogate for cervical cancer. ${ }^{28}$ Standard-directed biopsies found 30 women with CIN3+ and the DSI-assisted biopsies found another 15 , a relative gain of $50 \%$. The detection rate of standard-directed biopsy for finding CIN3+ among the 881 women was $3.41 \%$ (95\% CI: $2.31 \%-4.83 \%)$ and the detection rate of the DSI-assisted biopsies for CIN3+ among the remaining 851 women was $1.76 \%$ (95\% CI: $0.99 \%$ $2.89 \%$ ). This is a borderline statistically significant increase (two-sided $p=0.054$, exact binomial test).

Overall, there were 550 women with standard-directed and/or DSI-assisted biopsy/ies that were all $<\mathrm{CIN} 2$, so the rate of patients with negative biopsy/ies was $62.43 \%$ (95\% CI: $59.13 \%-65.62 \%)$. Among the 509 women who had standarddirected biopsy/ies, there were 431 women where standard biopsy/ies only found $<\mathrm{CIN} 2$, resulting in a negative biopsy rate of $48.92 \%$ ( $95 \%$ CI: $45.57 \%-52.28 \%$ ). Among the remaining 372 women who had no standard-directed biopsy, 131 had DSI-assisted biopsy/ies which were all <CIN2, resulting in a negative biopsy rate of $35.22 \%(95 \% \mathrm{CI}$ : $30.36 \%-40.31 \%)$. The DSI-assisted negative biopsy rate was significantly lower than the negative biopsy rate of standard-directed biopsy in the first phase of the examination (two-sided $p<0.0001$; exact binomial test).

The NND describes how many women underwent biopsy, which detected no CIN2+ for each woman who was found with CIN2+. This was 5.53 for standard-directed biopsies and 
Table 2 Total number of biopsies taken and numbers of patients with CIN2+/CIN3+ detected by type of biopsy, for different age groups, referral groups, and number of biopsies per patient

\begin{tabular}{|c|c|c|c|}
\hline \multirow[t]{2}{*}{ Patient group } & \multicolumn{3}{|l|}{ Type of biopsy } \\
\hline & Standard-directed & DSI-assisted & Random \\
\hline \multicolumn{4}{|l|}{ All patients $(\mathrm{n}=88 \mathrm{I})$} \\
\hline Number of biopsies taken & 808 & 365 & 16 \\
\hline Patients with CIN2+ & 78 & 34 & 1 \\
\hline Patients with CIN3+ & 30 & 15 & 1 \\
\hline \multicolumn{4}{|l|}{ Age $2 \mid-24$ years $(n=|| 0)$} \\
\hline Number of biopsies taken & 109 & 41 & 0 \\
\hline Patients with CIN2+ & 4 & 2 & 0 \\
\hline Patients with CIN3+ & I & 1 & 0 \\
\hline \multicolumn{4}{|l|}{ Age $25-29$ years $(n=185)$} \\
\hline Number of biopsies taken & 189 & 66 & 1 \\
\hline Patients with CIN2+ & 21 & 7 & 0 \\
\hline Patients with CIN3+ & 7 & 3 & 0 \\
\hline \multicolumn{4}{|l|}{ Age $\geq 30$ years $(n=586)$} \\
\hline Number of biopsies taken & 510 & 258 & 15 \\
\hline Patients with CIN2+ & 53 & 25 & 1 \\
\hline Patients with CIN3+ & 22 & 11 & 1 \\
\hline \multicolumn{4}{|l|}{ HSIL, ASC-H, AGC $(n=\mid I 0)$} \\
\hline Number of biopsies taken & 133 & 65 & 2 \\
\hline Patients with CIN2+ & 28 & 9 & 0 \\
\hline Patients with $\mathrm{CIN} 3+$ & 16 & 7 & 0 \\
\hline \multicolumn{4}{|c|}{ LSIL, ASC-US/HPV+, HPV+ $(n=77 I)$} \\
\hline Number of biopsies taken & 675 & 300 & 14 \\
\hline Patients with $\mathrm{CIN} 2+$ & 50 & 25 & 1 \\
\hline Patients with $\mathrm{CIN} 3+$ & 14 & 8 & I \\
\hline \multicolumn{4}{|l|}{ Patients with I biopsy $(n=3 \mid 6)$} \\
\hline Number of biopsies taken & 199 & 116 & 1 \\
\hline Patients with CIN2+ & 26 & 12 & 0 \\
\hline Patients with CIN3+ & 5 & 5 & 0 \\
\hline \multicolumn{4}{|l|}{ Patients with 2 biopsies $(n=2 \mid 0)$} \\
\hline Number of biopsies taken & 295 & 121 & 4 \\
\hline Patients with $\mathrm{CIN} 2+$ & 25 & 11 & 0 \\
\hline Patients with CIN3+ & 13 & 7 & 0 \\
\hline \multicolumn{4}{|c|}{ Patients with $>2$ biopsies $(n=|4|)$} \\
\hline Number of biopsies taken & 314 & 128 & 11 \\
\hline Patients with CIN2+ & 27 & 11 & 1 \\
\hline Patients with CIN3+ & 12 & 3 & 1 \\
\hline
\end{tabular}

Abbreviations: AGC, atypical glandular cells; ASC-H, atypical squamous cells (cannot exclude HSIL); ASC-US, atypical squamous cells of undermined significance; CIN, cervical intraepithelial neoplasia; DSI, dynamic spectral imaging; HPV, human papillomavirus; HSIL, high-grade squamous intraepithelial lesion; LSIL, low-grade squamous intraepithelial lesion.

3.85 for DSI-assisted biopsies. The PPV of standard-directed biopsies was $13.24 \%$ (95\% CI: $10.98 \%-15.78 \%)$, and of DSIassisted biopsies $16.16 \%$ (95\% CI: 12.54\%-20.35\%). These findings indicate that, despite the per-protocol "handicap" of coming second (after most obvious disease would have been identified), the additional DSI-assisted biopsies increased the efficiency to find CIN2+. This suggests that the higher detection of CIN2+ lesions also results from an improved

Table 3 Detection of patients with $\mathrm{CIN} 2+$, CIN3+, and $<\mathrm{CIN} 2$ per examination phase

\begin{tabular}{|c|c|c|c|c|c|c|}
\hline \multirow[t]{2}{*}{ Outcome } & \multicolumn{2}{|c|}{$\begin{array}{l}\text { Ist phase } \\
\text { (standard-directed biopsies) }\end{array}$} & \multicolumn{2}{|c|}{$\begin{array}{l}\text { Incremental 2nd phase } \\
\text { (DSI-assisted biopsies) }\end{array}$} & \multirow{2}{*}{$\begin{array}{l}\text { Significance } \\
p \text {-value }\end{array}$} & \multirow{2}{*}{$\begin{array}{l}\text { Relative gain } \\
\%\end{array}$} \\
\hline & $n(N)$ & $\%$ rate $(95 \% \mathrm{Cl})$ & $n(N)$ & $\%$ rate $(95 \% \mathrm{Cl})$ & & \\
\hline $\mathrm{CIN} 2+$ & $78(88 I)$ & $8.85(7.06-10.93)$ & $34(803)$ & $4.23(2.95-5.87)$ & 0.0001 & 43.6 \\
\hline CIN3+ & $30(88 \mathrm{I})$ & $3.4 I(2.3 I-4.83)$ & I5 (85I) & $1.76(0.99-2.89)$ & 0.054 & 50.0 \\
\hline$<\mathrm{CIN} 2$ & $43 I$ (88I) & $48.92(45.57-52.28)$ & 131 (372) & $35.22(30.36-40.31)$ & $<0.0001$ & $\mathrm{n} / \mathrm{a}$ \\
\hline
\end{tabular}

Notes: Women with no biopsies were not included; random biopsies and their outcomes were not considered.

Abbreviations: CIN, cervical intraepithelial neoplasia; DSI, dynamic spectral imaging; n, number of women; N, addressable women; n/a, not applicable. 
diagnostic ability when using the DSI map rather than solely because of the increased number of biopsies taken.

\section{Discussion}

DSI-assisted biopsies increased the detection rate for CIN2+ patients from $8.85 \%$ (standard-directed biopsies) to $12.71 \%$, a relative gain of $43.49 \%$. The detection increased also for CIN3+, across the different referral groups, and was more pronounced in women $\geq 30$ years old (Table 2), suggesting it is clinically relevant rather than, eg, regressive CIN2 lesions in younger women. ${ }^{29}$ The main strength of the study is that it collected multi-site "real world" data which reflect practice across US community-based clinics, as the use of the adjunctive DSI map by colposcopists was by clinical judgment, rather than prescribed. Using each patient as her own control for all comparisons, it confirms previous studies of $\mathrm{DSI}^{21-25}$ and demonstrates their applicability in a wider clinical setting and in a larger cohort of patients with sufficient statistical power.

Taking additional colposcopic biopsies increases disease detection, ${ }^{8,9}$ and since the number of biopsies was not controlled, the results if the participating colposcopists had followed an alternative multiple-biopsy protocol to add biopsies during the second phase rather than using the DSI map, can only be inferred by the efficiency of biopsy to find disease. Selection of biopsy during the initial phase (standard visualization), inevitably detected the most obvious disease, so biopsies taken during the second phase, would have to detect among subtler appearances and their efficiency to detect disease should drop. However, DSI used after biopsy selection by standard visualization was complete, improved the efficiency of biopsy to detect disease, which is consistent with the DSI map improving diagnostic ability rather than simply being a driver for more biopsies. Put another way, without using the DSI map, it would have taken a higher number of biopsies to achieve the same improved detection rate.

Furthermore, considering our data, and evidence from standard colposcopy practice from US community-based clinics, ${ }^{14}$ despite recommendations to biopsy every lesion, ${ }^{10}$ biopsy is still practiced conservatively and random biopsy is rare. So, in this setting, even if extra biopsies (without DSI) were requested by the protocol, as they are not part of standard practice, they may not have been taken and some CIN2+ patients would have remained undetected.

A possible limitation is that as clinicians had to make diagnostic decisions for the best interest of each patient, sometimes the DSI map may have been used not to add a biopsy, but to improve placement of a standard biopsy if clinically justified. However, in $65 \%$ of the women detected by DSI-assisted biopsies, upon completion of the first phase of the examination the colposcopists had confirmed that they did not intend to take a (standard) biopsy. This introduces uncertainty in the standard biopsy results for the remaining cases, as only the DSI-assisted biopsy was taken, but the associated improvement in efficiency suggests it is a justified implication of assessing "real world" use, with the benefit of detecting disease without increasing the number of biopsies, with the associated harms ${ }^{30}$ and costs.

In light of these results, it is worth discussing different recommendations to add biopsies with respect to the setting and biopsy efficiency, also considering whether the findings of institution-based studies translate to community-based practice. Wentzensen et $\mathrm{al}^{11}$ argue for taking multiple directed biopsies from each patient, by sampling all acetowhite areas, and by taking multiple samples from larger lesions to ensure the worst part is captured. Belinson and Pretorius propose a standard four-quadrant biopsy protocol, whereby after biopsying visualized lesions, any quadrants without visible lesions are biopsied at the squamocolumnar junction; ${ }^{13}$ Huh et al showed that, in some populations, a single random biopsy at the squamocolumnar junction when colposcopy is negative detects a significant number of CIN2+. ${ }^{31}$ A direct comparison of these results to this study's results is difficult as each study was conducted differently, potentially on populations of different background risk-levels and by colposcopists of varying levels of expertise. Random biopsies have been reported to have an efficiency ten times smaller than directed biopsies. ${ }^{9,12}$ Although biopsy efficiency is not reported by Wentzensen et al, ${ }^{11}$ the median number of biopsies among patients eventually found with $<$ CIN2 $(\sim 60 \%$ of the study population) was three, indicating a high number of biopsies that found no precancerous disease. This is in contrast to our analyses on biopsy efficiency which show that DSI-assisted biopsies did not compromise the trade-off between detection and unnecessary biopsying.

With each patient serving as her own control, bias due to patient selection or colposcopist variations is avoided. The inclusion of some women under 29 years, although their referral indication was not strictly meeting guidelines, ${ }^{1,19}$ has no effect on the results or conclusions $(<8 \%$ of the total CIN2+ was among them), and has the advantage that the study includes this age group as seen at US communitybased clinics.

The study assessed the pragmatic use of DSI and biopsying all DSI-positive areas was not imposed; consequently some disease although highlighted may have been missed. 
In a previous European research trial, ${ }^{23}$ colposcopists were required, by protocol, to biopsy the worst indication by DSI on each patient, and to also take one random biopsy per woman for verification of negatives. For comparison, in that setting, the sensitivity for patients with $\mathrm{CIN} 2+$ increased from $55 \%$ to $88 \%$ over the standard colposcopic assessment, with specificity dropping from $77 \%$ to $69 \%$.

\section{Conclusion}

In "real world" colposcopy, a significant portion of precancerous cervical disease is being missed despite evidence and guidance to improve detection. ${ }^{8-11,13,31}$ The results presented here confirm previous reports on using DSI mapping at colposcopy ${ }^{21-25}$ and show that in US community-based colposcopy, where the number of biopsies taken appears to be lower than in academic centers, ${ }^{14}$ the as-intended use of the DSI map increased the detection rate of high-grade CIN without compromising the proportion of patients with $<$ CIN2 biopsies. The impact of this result is an improved sensitivity to detect disease, timely management of women with pre-cancerous lesions and ultimately, improved cost-effectiveness. ${ }^{32}$

\section{Acknowledgment}

IMPROVE-COLPO was sponsored by DYSIS Medical, Edinburgh, UK.

\section{Disclosure}

JL, WRS, SAD and CGO: received speaker or advisory fees from DYSIS Medical outside the submitted work; PTL: received consulting fees from DYSIS Medical; EP: employee of DYSIS Medical. The authors report no other conflicts of interest in this work.

\section{References}

1. Saslow D, Solomon D, Lawson HW, et al. American Cancer Society, American Society for Colposcopy and Cervical Pathology, and American Society for Clinical Pathology Screening Guidelines for the Prevention and Early Detection of Cervical Cancer. J Low Genit Tract Dis. 2012; 16(3):175-204.

2. Massad LS, Collins YC. Strength of correlations between colposcopic impression and biopsy histology. Gynecol Oncol. 2003;89(3): 424-428.

3. ASCUS-LSIL Traige Study (ALTS) Group. Results of a randomized trial on the management of cytology interpretations of atypical squamous cells of undetermined significance. Am J Obstet Gynecol. 2003;188(6):1383-1392.

4. ASCUS-LSIL Traige Study (ALTS) Group. A randomized trial on the management of low-grade squamous intraepithelial lesion cytology interpretations. Am J Obstet Gynecol. 2003;188(6):1393-1400.

5. Jeronimo J, Schiffman M. Colposcopy at a crossroads. Am J Obstet Gynecol. 2006;195(2):349-353.
6. Massad LS, Jeronimo J, Katki HA, et al. The accuracy of colposcopic grading for detection of high-grade cervical intraepithelial neoplasia. J Low Genit Tract Dis. 2009;13(3):137-144.

7. Cantor SB, Cárdenas-Turanzas M, Cox DD, et al. Accuracy of colposcopy in the diagnostic setting compared with the screening setting. Obstet Gynecol. 2008;111(1):7-14.

8. Gage JC, Hanson VW, Abbey K, et al. Number of cervical biopsies and sensitivity of colposcopy. Obstet Gynecol. 2006;108(2):264-272.

9. Pretorius R, Belinson J, Burchette R, Hu S, Zhang X, Qiao YL. Regardless of skill, performing more biopsies increases the sensitivity of colposcopy. J Low Genit Tract Dis. 2011;15(3):180-188.

10. American College of Obstetricians and Gynecologists. Practice Bulletin No. 140: management of abnormal cervical cancer screening test results and cervical cancer precursors. Obstet Gynecol. 2013;122(6): 1338-1366.

11. Wentzensen N, Walker JL, Gold MA, et al. Multiple biopsies and detection of cervical cancer precursors at colposcopy. J Clin Oncol. 2015;33(1):83-89.

12. Pretorius RG, Belinson JL, Azizi F, Peterson PC, Belinson S. Utility of random cervical biopsy and endocervical curettage in a low-risk population. J Low Genit Tract Dis. 2012;16(4):333-338.

13. Belinson JL, Pretorius RG. A standard protocol for the colposcopy exam. J Low Genit Tract Dis. 2016;20(4):e61-e62.

14. Papagiannakis E, Huh WK. 2017 ASCCP Oral Presentation Abstracts. J Low Genit Tract Dis. 2017;21:S1-S17.

15. Monk BJ, Brewster WR. Does the ALTS trial apply to the communitybased practitioner? Am J Obstet Gynecol. 2003;188(6):1381-1382.

16. Stoler MH, Vichnin MD, Ferenczy A, et al. The accuracy of colposcopic biopsy: analyses from the placebo arm of the Gardasil clinical trials. Int J Cancer. 2011;128(6):1354-1362.

17. Wentzensen N, Zuna RE, Sherman ME, et al. Accuracy of cervical specimens obtained for biomarker studies in women with CIN3. Gynecol Oncol. 2009;115(3): 493-496.

18. Landers EE, Erickson BK, Bae S, Huh WK. Trends in colposcopy volume: where do we go from here? J Low Genit Tract Dis. 2016;20(4): 292-295.

19. Huh WK, Ault KA, Chelmow D, et al. Use of primary high-risk human papillomavirus testing for cervical cancer screening: interim clinical guidance. Gynecol Oncol. 2015;136(2):178-182.

20. van der Marel J, van Baars R, Quint WG, et al. The impact of human papillomavirus genotype on colposcopic appearance: a cross-sectional analysis. BJOG. 2014;121(9):1117-1126.

21. Soutter WP, Diakomanolis E, Lyons D, et al. Dynamic spectral imaging: improving colposcopy. Clin Cancer Res. 2009;15(5):1814-1820.

22. Louwers JA, Zaal A, Kocken M, et al. The performance of dynamic spectral imaging colposcopy depends on indication for referrals. Gynecol Oncol. 2015;139(3):452-457.

23. Louwers JA, Zaal A, Kocken M, et al. Dynamic spectral imaging colposcopy: higher sensitivity for detection of premalignant cervical lesions. BJOG. 2011;118(3):309-318.

24. Zaal A, Louwers JA, Berkhof J, et al. Agreement between colposcopic impression and histological diagnosis among human papillomavirus type 16-positive women: a clinical trial using dynamic spectral imaging colposcopy. BJOG. 2012;119(5): 537-544.

25. Coronado PJ, Fasero M. Colposcopy combined with dynamic spectral imaging. A prospective clinical study. Eur J Obstet Gynecol Reprod Biol. 2016;196:11-16.

26. Massad LS, Einstein MH, Huh WK, et al. 2012 updated consensus guidelines for the management of abnormal cervical cancer screening tests and cancer precursors. Obstet Gynecol. 2013;121(4):829-846.

27. Hiatt RA, Pasick RJ, Stewart S, et al. Community-based cancer screening for underserved women: design and baseline findings from the Breast and Cervical Cancer Intervention Study. Prev Med. 2001;33(3): 190-203.

28. Schiffman M, Rodríguez AC. Heterogeneity in CIN3 diagnosis. Lancet Oncol. 2008;9(5):404-406. 
29. Castle PE, Schiffman M, Wheeler CM, Solomon D. Evidence for frequent regression of cervical intraepithelial neoplasia-grade 2. Obstet Gynecol. 2009;113(1):18-25.

30. TOMBOLA (Trial Of Management of Borderline and Other Low-grade Abnormal smears) Group, Sharp L, Cotton S, After-effects reported by women following colposcopy, cervical biopsies and LLETZ: results from the TOMBOLA trial. BJOG. 2009;116(11):1506-1514.

31. Huh WK, Sideri M, Stoler M, Zhang G, Feldman R, Behrens CM. Relevance of random biopsy at the transformation zone when colposcopy is negative. Obstet Gynecol. 2014;124(4):670-678.
32. Wade R, Spackman E, Corbett M, et al. Adjunctive colposcopy technologies for examination of the uterine cervix - DySIS, LuViva Advanced Cervical Scan and Niris Imaging System: a systematic review and economic evaluation. Health Technol Assess. 2013;17(8):1-240.

International Journal of Women's Health

\section{Publish your work in this journal}

The International Journal of Women's Health is an international, peerreviewed open-access journal publishing original research, reports, editorials, reviews and commentaries on all aspects of women's healthcare including gynecology, obstetrics, and breast cancer. The manuscript management system is completely online and includes a very quick and fair peer-review system, which is all easy to use. Visit http://www.dovepress.com/testimonials.php to read real quotes from published authors.

\footnotetext{
Submit your manuscript here: http://www.dovepress.com/international-journal-of-womens-health-journal
} 